\title{
Time periodic solutions for the full quantum Euler equation
}

\author{
Min $\mathrm{LI}^{1}$ and Xianzhong Yao ${ }^{1}$ \\ ${ }^{1}$ Shanxi University of Finance and Economics
}

August 4, 2020

\begin{abstract}
In this paper, we establish the existence and uniqueness of a time periodic solution to the full compressible quantum Euler equations. First, we prove the existence of time periodic solutions under some smallness assumptions imposed on the external force in a periodic domain by a regularized approximation scheme and the Leray-Schauder degree theory. Then the result is generalized to $\$ \backslash \operatorname{mathbb}\{\mathrm{R}\}^{\wedge}\{3\} \$$ by adapting a limiting method and a diagonal argument. The uniqueness of the time periodic solutions is also given. Compared to classical Euler equations, the third-order quantum spatial derivatives are considered which need some elaborated treatments thereof in obtaining the highest-order energy estimates.
\end{abstract}

\section{Hosted file}

periodicsolutionLY.pdf available at https://authorea.com/users/348496/articles/473785-timeperiodic-solutions-for-the-full-quantum-euler-equation 